\title{
Role Of PET/CT in Assessment of Post Interventional Treatment of Hepatocellular Carcinoma
}

\author{
Heba Elmenshawy ${ }^{a}$, Ahmed Farid ${ }^{b}$, Hamada Khater ${ }^{\text {b }}$
}

\begin{abstract}
a Department of
Radiodiagnosis, Nasr city for health insurance hospital , Cairo, Egypt. ${ }^{\mathrm{b}}$ Department of Radiodiagnosis, Faculty of Medicine, Benha University hospitals, , Egypt
\end{abstract}

Correspondence to: Heba elmenshawy, Department of Radiodiagnosis, Nasr city for health insurance hospital, Cairo, Egypt

Email:

hebaelmenshawy7@gmail.com

Received: 14 April 2020

Accepted: 18 May2020

\begin{abstract}
:
Purpose: to highlight the role of PET/CT in evaluation of post interventional treatment (radiofrequency ablation \& TACE) of hepatocellular carcinoma. Background: Hepatocellular carcinoma (HCC) is one of the major causes of mortality among patients with cirrhosis. It represents about $11.85 \%$ of the malignancies of all GIT organs and $1.78 \%$ of the total malignancies among Egyptians. Patients and methods: From February 2018 to November 2019, twenty five patients (20 males and 5 females, their age ranged from 44 to 75 years with mean age of $60.60 \pm 7.35$ years) were referred to the radiology unit with hepatocellular carcinoma and had underwent TACE ( 2 patients had history of combined TACE and RFA procedures ). ${ }^{18} \mathrm{~F}$ FDG PET/CT and triphasic CT examinations were conducted in a private radiology center in Cairo for all patients using combined PETCT machine (Philips intgenuity TF PET/CT 128 slices). Results: PET/CT showed true positive results in 20 patients, true negative results in 3 patients, false negative results at one patient and false
\end{abstract} positive results at one patient. In our study, the sensitivity, specificity, positive predictive value (PPV), negative predictive value (NPV) and accuracy of ${ }^{18} \mathrm{~F}$-FDG PET/CT were 95.24\%, 75\%, 95.24\%, 75\% and 92\% respectively. Conclusion: PET/CT showed higher accuracy in assessment of the intervention bed without affection by lipiodol artifact as well as detection of extra hepatic spread of HCC in single whole body examination which is crucial for patients preparing for liver transplantation and differentiation between benign and malignant portal vein thrombosis.

Key words: 18 F-FDG PET/CT, HCC, TACE, AFP 

Abbreviations:

\begin{tabular}{|c|c|}
\hline AFP & Alpha-fetoprotein \\
\hline CECT & $\begin{array}{l}\text { Contrast enhanced } \\
\text { tomography }\end{array}$ \\
\hline PET & Positron Emission Tomography \\
\hline CT & Computed tomography. \\
\hline CTAC & $\begin{array}{l}\text { Computed tomography } \\
\text { correction }\end{array}$ \\
\hline FDG & Fluorodeoxyglucose \\
\hline
\end{tabular}

\section{Introduction}

Hepatocellular carcinoma (HCC) represents the commonest primary hepatic tumor of adults. It is the 6th most common tumor in the world and the third commonest cause of cancer related deaths (1).

Based on their gene expression profiles HCCs with high ${ }^{18}$ F-FDG uptake are reported to be more aggressive than $\mathrm{HCCs}$ with low ${ }^{18}$ F-FDG uptake. Moreover, baseline PET/CT has great prognostic value for $\mathrm{HCC}$ patients who are treated with radiofrequency ablation and TACE $(2,3)$.

Interventional treatment has been applied to patients with inoperable HCC. Despite initial remission of $\mathrm{HCC}$ after interventional treatment, recurrence is common.

Since patients with recurrent HCC may be amenable to potentially curative resection, early detection of intrahepatic recurrence and /or extra hepatic metastases is extremely important and can facilitate successful retreatment at an early stage (4)

\section{Aim of the Work}

The aim of this study is to highlight the role of PET/CT in evaluation of post interventional treatment (radiofrequency ablation \& TACE) of hepatocellular carcinoma, hence guiding the clinician to proper management strategy

\section{Patients and Methods}

\section{$\underline{\text { Patients }}$}

The study was prospectively on twenty five patients (20 males and 5 females) during the period from February 2018 to November 2019, their age ranged from 44 to 75 years with mean age of $60.60 \pm 7.35$ years) were referred to the radiology unit with hepatocellular carcinoma and had underwent TACE ( 2 patients had history of combined TACE and RFA procedures ). ${ }^{18} \mathrm{~F}-\mathrm{FDG}$ $\mathrm{PET} / \mathrm{CT}$ and triphasic CT examinations were conducted in a private radiology center in Cairo for all patients using combined PETCT machine (Philips intgenuity TF PET/CT 128 slices). The study was approved by the institutional ethics committee.

\section{Inclusion criteria:}

- Patients of any age who underwent TACE for HCC.

- One patient had submitted to TACE on transplanted liver was included .

- Both sexes were included.

\section{Exclusion criteria:}

- Patients with past history of contrast 
allergy.

- Patients with blood glucose level $>300$ $\mathrm{mg} / \mathrm{dl}$ at the time of the study.

- High serum creatinine $>2 \mathrm{mg} / \mathrm{dl}$.

- Small lesions $<10 \mathrm{~mm}$

\section{Technique of examination:}

\section{Patient preparation:}

\section{2 days prior to examination:}

$\bigcirc$ Refrain from all strenuous activity.

\section{1 day prior to examination:}

- Limitation of carbohydrate diet for the previous 24 hours before the date of examination.

o High water intake was allowed.

\section{2 hours prior to examination:}

O Nothing to eat or drink, except water, for 6 hours before the examination. Drinking water as much as possible was needed until arrival.

o Serum creatinine level measurement.

O For diabetic patients:

* Patients on oral hypoglycemics must be fasting for at least 6 hours prior to the examination with administration of the routine oral medical regimen.

Patients on insulin regimen should control blood glucose level to be less than $200 \mathrm{mg} / \mathrm{dl}$ for at least 2 days prior to the examination, then patients had breakfast at the early morning with administration of the regular insulin regimen followed by total fasting, with exception of water intake.

\section{On the day of study:}

O Patient must be present 15-30 minutes before PET scan.

O A consent form was signed by the patient (or his guardian) after the detail of the procedure was explained.

O Remove metallic items and jewelry and the patient was given a gown to wear.

O Assessment of patient's body weight.

Serum glucose level was measured in the center just before 18F-FDG injection, and it must be less than $200 \mathrm{mg} / \mathrm{dl}$.

冈In case of diabetic patients with blood glucose level above 200 $\mathrm{mg} / \mathrm{dl}$, subcutaneous insulin injection was administered with monitoring of the blood glucose level until reaching the accepted level $(100-200 \mathrm{mg} / \mathrm{dl})$ then the study was started.

O Fixation of intravenous cannula.

O Detailed explanation of the procedure, including strict instructions to keep 
calm and avoid muscular activity prior to the examination.

FDG was administered and must circulate for approximately 50- 60 minutes prior to the scan, during this period; patient must be in quite resting position.

The scan took 20 minutes at the machine.

\section{1- 18 FDG Dose:}

○ $1 \mathrm{mCi} / 10 \mathrm{Kg}$ of $18 \mathrm{FDG}$ was administered to each patient 60 minutes before the examination.

\section{2- Examination time:}

The whole study took approximately 80 minutes (60 minutes in waiting room after FDG injection then 20 minutes at the PET /CT machine.

The examination was performed starting by low dose non enhanced CT scan for attenuation correction, then a whole body PET study followed by Triphasic CT.

\section{3- Patient position:}

$\checkmark$ The patient was asked to empty the bladder before entering the PET/CT machine.

$\checkmark$ The patient was introduced to the PETCT machine, lying in supine position with head fixation and arms elevated up.

\section{4- PET Technique:}

PET was performed following the attenuation correction CT study without moving the patient. Approximately 9 to 11 bed positions were planned in the three-dimensional acquisition mode for scanning the entire patient in one and half minute for image acquisition at each bed position.

\section{5- PET/CT Fusion:}

For each of these sets of PET and CT images, corresponding "fusion" images, combining the two types of data, were processed by special software to produce functional and anatomical image simultaneously.

\section{Results}

Twenty five patients (20 males and 5 females) were included in this study. Their age ranged from 44 to 75 years with mean age of $60.60 \pm 7.35$ years. All patients had history of local treatment of HCC by transarterial chemoembolization ( 2 patients had history of combined TACE and RFA)

The diameter of the lesions ranged between $1 \mathrm{~cm}$ and $8.5 \mathrm{~cm}$ with mean diameter of 4.2 $\mathrm{cm} \pm 1.79$.

The hepatic segmental distribution of the chemo-mobilized lesions was as following: 
segment II ( 1 patient), segment III ( 2 patients ), segment IV ( 2 patients), segment V ( 3 patients ), segment VI ( 3 patients ), segment VII ( 7 patients ), segment VIII ( 6 patients ) and one patient on top of transplanted liver (1 patient ) as described in table 1.

\begin{tabular}{|l|c|c|}
\hline \multicolumn{1}{|c|}{} & Total no. = 30 \\
\hline \multirow{3}{*}{ Site of lesions } & II & $1(4 \%)$ \\
& III & $2(8 \%)$ \\
IV & $2(8 \%)$ \\
& V & $3(12 \%)$ \\
& VI & $3(12 \%)$ \\
VII & $7(28 \%)$ \\
VIII & $6(24 \%)$ \\
Size of lesion & Transplanted liver & $1(4 \%)$ \\
& Mean \pm SD & $4.20 \pm 1.79$ \\
& Range & $1-8.5$ \\
\hline
\end{tabular}

To determine the accuracy of the results, our standard included laboratory serial AFP level monitoring, follow up by different imaging modalities and histopathological results of the resected liver in case of transplantation .

Serial AFP monitoring of the 25 patients were as following; 23 patients had persistent elevated AFP levels and the remaining 2 patients had normal levels of AFP and were preparing for liver transplantation. Out of the 23 patients with elevated AFP levels, triphasic CT study was positive for viable tumoral tissue in only 18 patients and showed no enhancement in 5 patients, among these CT negative patients, PET /CT showed positive nodular FDG uptake at intervention bed in 2 patients and the other 3 patients showed extra hepatic metastatic spread in spite of successful local chemoembolization. While PET/CT was positive in 22patients out of the 23 patients with elevated AFP levels, this leaved a single fore mentioned patient by PET/CT showed characteristic enhancement pattern of viable $\mathrm{HCC}$ at triphasic CT.

The other 2 patients with normal AFP level showed no abnormal findings on CT, yet on PET/CT there was a single false positive case due to regional hyperemia causing increased FDG uptake. This case of regional hyperemia underwent liver transplantation and histopathological results of the resected liver revealed complete necrosis with no malignant cells.

Among the patients with positive extra hepatic metastasis detected by PET/CT; one patient showed local recurrence at the intervention bed with metastatic abdominal lymph nodes and 2 patients were positive for extra hepatic metastasis in spite of successful local treatment ( one patient was positive for osseous deposits and the other one for supra renal metastasis).

PET/CT showed true positive results in 20 patients, true negative results in 3 patients, false negative results at one patient and false positive results at one patient. 
In our study, the sensitivity, specificity, positive predictive value (PPV), negative predictive value (NPV) and accuracy of ${ }^{18} \mathrm{~F}$ FDG PET/CT were 95.24\%, 75\%, 95.24\%, $75 \%$ and $92 \%$ respectively as described in table 2 .

\begin{tabular}{|c|c|c|c|c|c|}
\hline PET & Sensitivity & Specificity & PPV & NPV & Accuracy \\
\hline & $95.24 \%$ & $75 \%$ & $95.24 \%$ & $75 \%$ & $92 \%$ \\
\hline
\end{tabular}

PPV positive predictive value.

NPV negative predictive value

\section{Discussion}

Hepatocellular carcinoma is one of the most common malignant tumors worldwide. Early detection and treatment of recurrent $\mathrm{HCC}$ after loco regional interventional treatment are important for patient survival (2).

Percutaneous techniques such as TACE are now available to manage HCC. Assessment of tumor response after TACE is important to determine whether the tumor is completely eradicated or needs additional treatment (2).

CT and MRI are the most widely used tools to assess patients underwent locoregional intervention procedures such as TACE based on reduction in size or changes in the internal structure as well as enhancement pattern (2).

The evaluation of treatment response using size criteria, based on the Response Evaluation Criteria in Solid Tumors (RECIST), does not apply well post chemoembolization of HCC. So, most investigators have depended on the presence or absence of contrast enhancement for determination of therapeutic response (2).

The European Association for the Study of the Liver (EASL) criteria and the modified Response Evaluation Criteria in Solid Tumors (mRECIST) have been widely used for post TACE assessment however evaluation of the lesions based on enhancement criteria might not be in agreement with the actual biological assessment(5).

The beam-hardening artifact of highattenuation lipiodol on CT masks the intralesional viable tumor detection, in addition after TACE the feeding arteries of the residual tumor are significantly thinner; these changes may affect the degree of enhancement of the tumor (4).

The complete and homogenous intralesional deposition of lipiodol would indicate a high degree of necrosis within the lesion: however, it is difficult to discriminate correctly the viability and necrosis of the tumors in case of dispersed deposition of lipiodol, because lipiodolnegative areas do not correspond to viable 
areas of the tumors. On the contrary, wholebody 18 F-FDG PET/CT scan may represent a valuable imaging tool to detect residual viable tissue based on metabolic activity without affection by lipiodol artifact (4) as described in figure1.

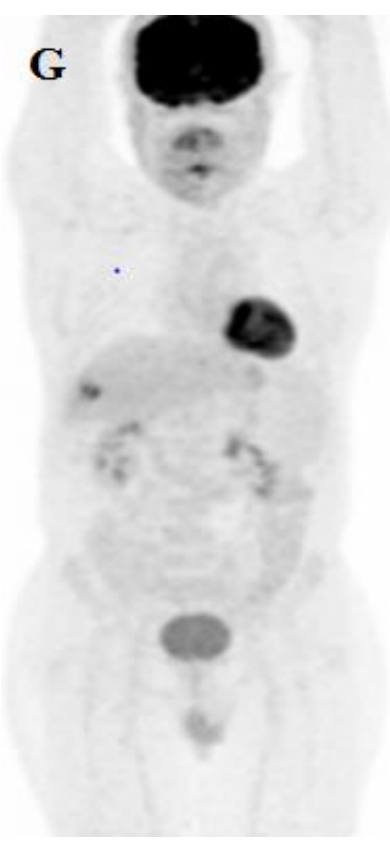

PET/CT is a unique combination of the cross-sectional anatomic data provided by CT and the metabolic data provided by PET. It has the advantage of local therapy assessment as well as detection of extra hepatic spread of HCC which is crucial for planning of liver transplantation. In contrast to the morphological image diagnosis, FDGPET evaluates viability based on glucose metabolism and it is not influenced by tumor morphology or lipiodol deposition (6) as described in figure2.

\section{H}
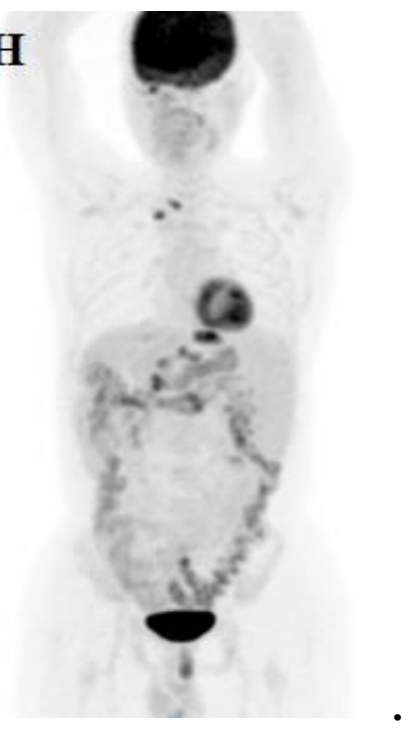

The avidity of FDG uptake was defined in our study as maximum standardized uptake value (SUV max). Positive FDG uptake is considered when the lesion uptake is higher than the physiological background activity of normal liver tissue which was also adopted by the study done by (7).

In our study the median value of TumorSUVmax in the positive cases was 7.4 (ranged from 3.5 to 24), most of them were poorly differentiated $\mathrm{HCCs}$, yet a single case measuring about 1.4 SUVmax was a well differentiated HCC type. In the study done previously (8) the median value of TumorSUVmax 4.3 (ranged from 2.0 to 11.6).

The median value of TSUV max / LSUV max in our study is 2.9 (range from 1.06 to 7.27) considering the cut off value 1.06 which is correlated to the study done before 
(9), where the TSUV max/LSUV max cut off value was 1.65 .

The false positive interpretations in PET/CT are due to physiological uptake, infection and recent chemotherapy whereas false negative results may be found with well differentiated $\mathrm{HCC}$ and the small lesions below the scanner resolution $(<10 \mathrm{~mm})(\mathbf{6})$. Therefore in our study the examination was done at least 6 weeks after TACE for the most accurate characterization of locoregional response after chemoembolization.

Small lesions $<10 \mathrm{~mm}$ that are below the scanner resolution might be missed unless they show avid FDG uptake on top of normal liver tissue background activity so lesions less than $10 \mathrm{~mm}$ were excluded from our study.

The diameter of the lesions in our work ranged between $1 \mathrm{~cm}$ and $8.5 \mathrm{~cm}$ (with mean diameter of $4.2 \mathrm{~cm}$ ) which is comparable to the study done by (8) where the diameter of the lesions ranged between 2.5 and $10.5 \mathrm{~cm}$ (with mean diameter of $5.5 \mathrm{~cm}$ ).

Although PET-CT has been found to show suboptimal sensitivity in detection of well differentiated HCC where the lesion FDG uptake is similar to the surrounding normal liver activity, it is useful in the detection of extra hepatic spread of HCC with superior accuracy in comparison with CT and MRI. According to (10), ${ }^{18}$ F-FDG PET has been used in many institutes to assess extra hepatic metastasis before liver transplantation for HCC.

The higher sensitivity of PET for detecting extra hepatic metastases of $\mathrm{HCC}$ as compared with primary lesions could be explained by an increased likelihood for metastasis to occur in poorly differentiated type of HCC, which tends to have a higher level of FDG uptake (11).

Our study demonstrated higher sensitivity of $\mathrm{PET} / \mathrm{CT}$ in detection of local viable tumoral tissue following TACE which may be masked by lipiodol artifact in addition to its ability to detect extra hepatic metastasis of HCC in a single whole body examination which may be crucial for patients preparing for liver transplantation hence influencing patient selection.

In our study PET/CT showed sensitivity, specificity and accuracy of $95.24 \%, 75 \%$ and $92 \%$ respectively. These results are comparable to many studies as (9) who reported that PET /CT sensitivity, specificity and accuracy for detection of viable HCC after TACE were $89.29 \%, 65.71 \%$ and $80.22 \%$ respectively. Also (2) stated that the respective values for sensitivity, specificity and accuracy of PET/ CT in the evaluation 
of early treatment response after interventional therapy for hepatocellular carcinoma were $87.5 \%, 71.4 \%$ and $80.0 \%$. Also (12) stated that the sensitivity and specificity of PET/ CT in assessment of post therapeutic hepatocellular carcinoma were $100 \%$, and $83.3 \%$ respectively and he stated that the relatively high sensitivity was attributed to that most positive $\mathrm{HCC}$ cases in his study were of poorly differentiated type which showed FDG avidity.

\section{References}

1- Dai L, Ren P, Liu M, Imai H, Tan EM and Zhang JY. Using immunomic approach to enhance tumor-associated autoantibody detection in diagnosis of hepatocellular carcinoma. Clin Immunol. (2014); 152: 127 139.

2- Kim SH, Won KS, Choi BW, JO I, Zeon SK, Chung WJ, et al. Usefulness of F-18 FDG PET/CT in the Evaluation of Early Treatment Response after Interventional Therapy for Hepatocellular Carcinoma. Nucl Med Mol Imaging. (2012); 46: 102-110.

3- Song MJ, Bae SH, Lee SW, Song DS, Kim HY, Yoo IR, et al. 18F-Fluorodeoxyglucose PET/CT predicts tumour progression after transarterial chemoembolization in hepatocellular carcinoma. Eur J Nucl Med Mol Imaging. (2013); 40: 865-873.

4- Sun L, Guan YS and Pan WM. Metabolic restaging of hepatocellular carcinoma using whole body 18 F FDG PET/CT. World J Hepatology. (2009); 1 (1): 90-97.

5- Shim JH, Lee HC and Kim SO. Which response criteria best help predict survival of patients with hepatocellular carcinoma following chemoembolization? A validation study of old and new models. Radiology. (2012); 262: 708-18.

6- Tsurusaki M, Okada M, Kuroda H, Mastsuki M, Ishii K and Murakami T. Clinical application of 18F-fluorodeoxyglucose positron emission tomography for assessment and evaluation after therapy for malignant hepatic tumor. J Gastroenterol. (2014); 49: 46-56.

7- Abuodeh $Y$, Naghavi AO and Ahmed KA. Prognostic value of pre-treatment F-18-FDG PET-CT in patients with hepatocellular carcinoma undergoing radioembolization. World J Gastroenterol. (2016); 22(47): 1040610414

8- Ahn SG, Kim SH and Jeon TJ. The role of preoperative $\left[{ }^{18} \mathrm{~F}\right]$ fluorodeoxyglucose positron emission tomography in predicting early recurrence after curative resection of hepatocellular carcinomas. J Gastrointest Surg. (2011) Nov; 15(11):2044-52.

9- Song HJ, Cheng JY, HU SL, Zhang GY, FU $Y$ and Zhang YJ. Value of 18F-FDG PET/CT in detecting viable tumor and predicting prognosis of hepatocellular carcinoma after TACE. Clinical Radiology. (2015); 70. 128e137.

10- Mocherla B, Kim J and Roayaie S. FDG $\mathrm{PET} / \mathrm{CT}$ imaging to rule out extrahepatic metastases before liver transplantation. Clin Nucl Med. (2007); 32: 947-948.

11- Wong SC, Ngai WT and Choi FPT. Update on Positron Emission Tomography for Hepatocellular Carcinoma. Hong Kong J Radiol. (2017); 20:192-204.

12- Soliman SS, Omar OH, Abo Elmaaty $M E$ and Ibrahim AM . Role of PET /CT in 
Assessment of Post Therapeutic Hepatocellular

Carcinoma. The Egyptian Journal of Hospital
Medicine (2017) October; Vol. 69 (4), Page 2271-2277.

To cite this article: Heba elmenshawy, Ahmed Farid, Hamada Khater. Role Of PET/CT in Assessment Of Post Interventional Treatment Hepatocellular Carcinoma. BMFJ 2021; 38 (Radiology):54-63. DOI: 10.21608/bmfj.2020.27937.1248 